\title{
Expansion of a SARS-CoV-2 Delta variant with an 872 nt deletion encompassing ORF7a, ORF7b and ORF8, Poland, July to August 2021
}

Natalia Mazur-Panasiuk ${ }^{1, *}$, Lukasz Rabalsk ${ }^{\mathrm{i},{ }^{\star}}$, Tomasz Gromowski ${ }^{1{ }^{1,3}}$, Grzegorz Nowicki ${ }^{4}$, Michal Kowalski ${ }^{5}$, Witold Wydmanski ${ }^{5}$ , Piotr Szulc ${ }^{5}$, Maciej Kosinski², Karolina Gackowska ${ }^{2}$, Natalia Drweska-Matelska ${ }^{4}$, Jakub Grabowski ${ }^{4}$, Anna PiotrowskaMietelska ${ }^{6}$, Boguslaw Szewczyk ${ }^{2}$, Krystyna Bienkowska-Szewczyk ${ }^{7}$, Jakub Swadzba ${ }^{6}$, Pawel Labaj $^{5}$, Maciej Grzybek $^{8}$, Krzysztof Pyrc ${ }^{1}$

1. Virogenetics Laboratory of Virology, Malopolska Centre of Biotechnology, Jagiellonian University, Krakow, Poland

2. Laboratory of Recombinant Vaccines, Intercollegiate Faculty of Biotechnology of University of Gdansk and Medical University of Gdansk, Gdansk, Poland

3. Human Genome Research Variation Group, Malopolska Centre of Biotechnology, Jagiellonian University, Krakow, Poland

4. genXone SA, Research \& Development Laboratory, Suchy Las, Poland

5. Bioinformatics Research Group, Malopolska Centre of Biotechnology, Jagiellonian University, Krakow, Poland

6. Diagnostyka Medical Laboratories, Krakow, Poland

7. Laboratory of Virus Molecular Biology, Intercollegiate Faculty of Biotechnology of University of Gdansk and Medical University of Gdansk, Gdansk, Poland

8. Department of Tropical Parasitology, Institute of Maritime and Tropical Medicine, Medical University of Gdansk, Gdynia, Poland

* These authors contributed equally to this article and share first authorship

Correspondence: Krzysztof Pyrc (k.a.pyrc@uj.edu.pl)

Citation style for this article:

Mazur-Panasiuk Natalia, Rabalski Lukasz, Gromowski Tomasz, Nowicki Grzegorz, Kowalski Michal, Wydmanski Witold, Szulc Piotr, Kosinski Maciej, Gackowska Karolina, Drweska-Matelska Natalia, Grabowski Jakub, Piotrowska-Mietelska Anna, Szewczyk Boguslaw, Bienkowska-Szewczyk Krystyna, Swadzba Jakub, Labaj Pawel, Grzybek Maciej, Pyrc Krzysztof. Expansion of a SARS-CoV-2 Delta variant with an 872 nt deletion encompassing ORF7a, ORF7b and ORF8, Poland, July to August 2021. Euro Surveill. 2021;26(39):pii=2100902. https://doi.org/10.2807/1560-7917.ES.2021.26.39.2100902

Routine genomic surveillance on samples from COVID19 patients collected in Poland during summer 2021 revealed the emergence of a SARS-CoV-2 Delta variant with a large 872 nt deletion. This change, confirmed by Sanger and deep sequencing, causes complete loss of ORF7a, ORF 7 , and ORF8 genes. The index case carrying the deletion is unknown. The standard pipeline for sequencing may mask this deletion with a long stretch of N's. Effects of this deletion on phenotype or immune evasion needs further study.

Between 1 July and 16 August 2021, 316 severe acute respiratory syndrome coronavirus 2 (SARS-CoV-2) genomes from 13 of 16 Polish voivodships from isolates obtained during routine surveillance activities, were deposited by our group in the GISAID database [1]. Of these, 31 sequences, all of which belonged to the SARSCoV-2 variant of concern (VOC) Delta (Phylogenetic Assignment of Named Global Outbreak (Pango) lineage designation B.1.617.2) AY.4 sublineage designation, showed an unusual pattern i.e. a long stretch of N's, indicating a weak or undetermined nucleotide signal, in the region corresponding to the open reading frame (ORF) $7 a, O R F 7 b$ and ORF8 genes in the reference SARSCoV-2 genome (GenBank NC_045512).

Most of these unique sequences (27/31) originated from coronavirus disease (COVID-19) patient samples collected in the Malopolska voivodship in southern Poland, between 17 July and 11 August 2021. Our aim was to investigate the emergence of the unusual sequence pattern in SARS-CoV-2 sequences from Poland.

\section{Clinical presentation}

Clinical data were available for 24 of the 31 patients with atypical Delta VOC sequences. COVID-19 was suspected in all patients, since they showed clinical symptoms compatible with the disease; all were confirmed by a SARS-CoV-2 RT-qPCR diagnostic test, referred by a general practitioner. The median age was 31 years (range: 4 months-84 years), with an equal number of females $(n=12)$ and males $(n=12)$. Symptoms included fever $(n=23)$, cough $(n=15)$, headache $(n=10)$, weakness $(n=10)$, muscle pain $(n=10)$, runny nose $(n=7)$, loss of smell $(n=3)$, loss of taste $(n=2)$, sore throat $(n=1)$, diarrhoea $(n=1)$, dyspnoea $(n=1)$ and breathing difficulties $(n=1)$. Seven patients were hospitalised, and four needed oxygen supplementation. Fifteen of the 24 patients were not vaccinated or partially vaccinated (one dose, various mRNA and non-mRNA vaccines) against SARS-CoV-2; of the seven hospitalised patients, five were not vaccinated. None of the patients travelled abroad before the COVID-19 symptoms developed. 
Primer pairs used for amplification and Sanger sequencing of selected SARS-CoV-2 specimens, Poland, 17 July-11 August 2021

\begin{tabular}{|c|c|c|c|}
\hline Pair & Sequencing protocol & Primer name & Primer sequence ( $5^{\prime}$ to $\left.3^{\prime}\right)$ \\
\hline \multirow{2}{*}{ Pair 1} & \multirow{2}{*}{ ARTICv3 } & 90_Left & ACACAGACCATTCCAGTAGCAGT \\
\hline & & 93_Right & AGGTCTTCCTTGCCATGTTGAG \\
\hline \multirow{2}{*}{ Pair 2} & \multirow{2}{*}{ Midnight } & 27_Left & TGGATCACCGGTGGAATTGCTA \\
\hline & & 28_Right & GTTTGGCCTTGTTGTTGTTGGC \\
\hline
\end{tabular}

SARS-CoV-2: severe acute respiratory syndrome coronavirus 2.

ARTICv3 [4,5] and Midnight protocols [3] are whole genome sequencing procedures based on amplification of specific SARS-CoV-2 genome regions with the use of sets of primers.

\section{PCR and sequencing analysis}

For sample collection, nasopharyngeal swab samples were taken according to the WHO guidelines and in line with the protocols approved by the Polish Ministry of Health [2]. RNA was extracted by the various laboratories, accordingly to their validated, routinely performed methods used for SARS-CoV-2 diagnostic. Extracted RNA was selected randomly, was frozen at $-80^{\circ} \mathrm{C}$ and sent from collection to sequencing laboratories within 2 weeks. The sequencing was performed with Oxford Nanopore Technologies (ONT) platform (Oxford, United Kingdom) using the ARTIC amplicon v3 and Midnight whole genome sequencing protocols [3-5]. Sequencing results showed a long stretch of N's within the region covering the ORF7a, ORF7b and ORF8 genes. Since the technique is based on the targeted amplification, this observed artefact i.e. a long stretch of N's, was suspected to be the result of a short insertion-deletion mutation (indel) or single nucleotide polymorphism (SNP) within the primer target sites. We selected 14 specimens for further genetic analyses. To exclude an unspecific primer binding event, we tested the primer pair (e.g. 90_Left and 93_Right primers, which was used for the ARTICv3 protocol and overlapped the region of interest) for SARS-CoV-2 genome amplification (Table 1).

Agarose gel electrophoresis showed that the amplification products obtained from the ARTICV3 protocol primers $[4,5]$ were ca $500 \mathrm{bp}$, rather than the expected size of $1,328 \mathrm{bp}$ (Figure 1).

This unexpected result suggested a large ca850 nt deletion in these isolates. In order to exclude mispriming events, the second pair of primers originating from the Midnight protocol [3] (e.g. 27_Left, 28_Right) (Table 1)(Figure 1) was used to confirm the deletion. Similar to the ARTICV3 primer pair, the products of the second primer set were ca $850 \mathrm{nt}$ shorter than the expected 2,265 bp.

The obtained amplicons were subjected to in-house ONT sequencing using native barcoding for the whole amplicon. In parallel, the products obtained in the first round of RT-PCR were subjected to Sanger sequencing. Both methods confirmed a deletion of $872 \mathrm{nt}$, which stretches between nucleotide positions 27,385 and 28,256 of the reference genome. This sizable alteration causes a complete loss of ORF $7 a, O R F 7 b$, and ORF8 genes (Figure 2). The obtained sequences were deposited in the GISAID database under the following accession numbers: hCoV-19/Poland/PL_ P3686/2021; hCoV-19/Poland/PL_P3688/2021; hCoV19/Poland/PL_P3693/2021; hCoV-19/Poland/ P L_P $3695 / 2021$; h CoV-19/Poland / P L P3696/2021; hCoV-19/Poland/PL_P3699/2021; hCoV19/Poland/PL_P3704/2021; hCoV-19/Poland/ PL_P3705/2021; h CoV-19/Poland/PL P3709/2021; hCoV-19/Poland/PL_P3711/2021; hCoV19/Poland/PL_P3794/2021; hCoV-19/Poland/ PL_P3804/2021; hCoV-19/Poland/PL_P3687/2021; hCoV-19/Poland/PL_P3710/2021.

\section{Rapid barcoding error exclusion}

Such methods of high-throughput whole genome sequencing like ONT of SARS-CoV-2 are usually performed in a 96-well plate format using the barcoding approach, which is time- and cost-effective, but may lead to sporadic misclassification of some reads between the samples. This mistake could be wrongly reported as 'quasi-species'. To exclude artefacts related to the barcoding procedure and to detect SARSCoV-2 quasi-species, selected samples were run individually on a single flow cell. Moreover, during our analyses, we used a highly accurate ONT Guppy 5.0.11 super high accuracy (SUP) base-calling algorithm supported by detecting a barcode from both ends. None of the reads were mapped to the deleted region, which supported the hypothesis that isolates containing the 872 nt deletion are not mixed with the wild-type virus, thus representing a pure population of these new Delta AY.4 isolates with the 872 nt deletion.

\section{Viral load}

The viral load, expressed as a mean cycle threshold (CT) value obtained during diagnostic RT-qPCR, was available for 30 samples for three different commonly used gene targets ( $E$ : envelope gene, $N$ : nucleoprotein gene, ORF1a: ORF1a gene). To assess the potential influence of the $872 \mathrm{nt}$ deletion on the virus transmissibility, the CT was compared with selected other Delta variant AY.4 lineage samples $(n=34)$ confirmed in Poland between 15 July and 16 August 2021 (Figure 3). The viral loads did not vary between the groups, and 
Amplified PCR fragments from isolates expressing a unique SARS-CoV-2 Delta variant sequence, Poland, 17 July-11 August $2021(\mathrm{n}=14)$

A.

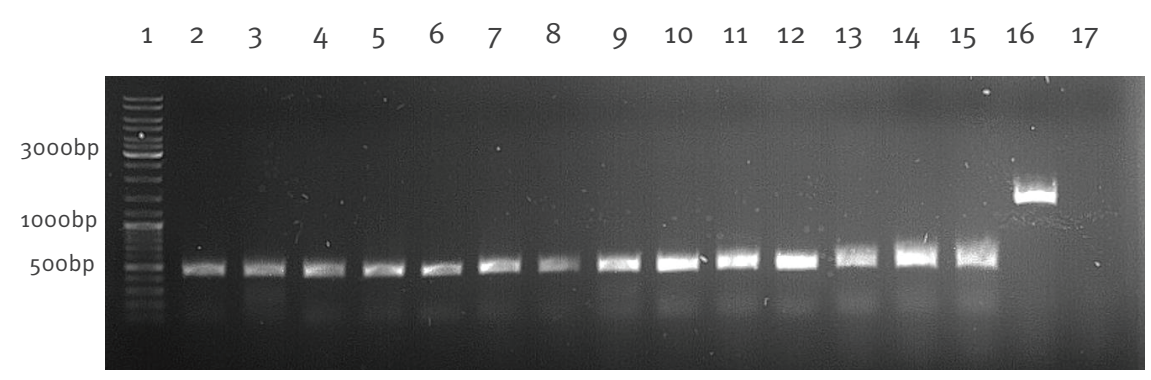

B.
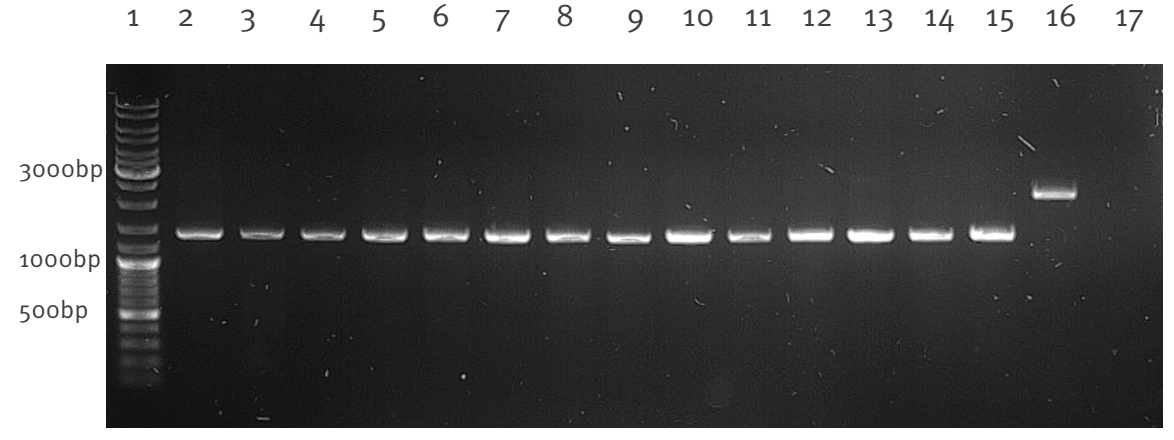

SARS-CoV-2: severe acute respiratory syndrome coronavirus 2.

PCR was performed with (A) ARTICv3 primers (90_Left, 93_Right) [4,5] and (B) Midnight primers (27_Left, 28_Right) [3] and analysed with agarose gel electrophoresis. Isolates with unique SARS-CoV-2 Delta variant sequences (lanes 2-15) reveal a band at ca $500 \mathrm{bp}(\mathrm{A})$ and at ca $1400 \mathrm{bp}(\mathrm{B})$ rather than the expected size of 1,328 bp (lane 16, A) or 2,265 bp (lane 16, B). Lane 1: GeneRuler DNA ladder mix (Thermo Fisher Scientific); Lane 2: sample hCoV19/Poland/PL_P3804/2021; Lane 3: sample hCoV-19/Poland/PL_P3711/2021; Lane 4: sample hCoV-19/Poland/ PL_P3699/2021; Lane 5: sample hCoV-19/Poland/PL_P3705/2021; Lane 6: sample hCoV-19/Poland/PL_P3687/2021; Lañe 7: sample hCoV-19/Poland/PL_P3686/2021; Lane 8: sample hCoV-19/Poland/PL_P3695/2021; Lane 9: sample hCoV-19/Poland/PL P3696/2021; Lane 10: sample hCoV-19/Poland/PL_P3688/2021; Lane 11: sample hCoV-19/ Poland/PL_P3693/2021; Lane 12: sample hCoV-19/Poland/PL_P3704/2021; Lane 13: sample hCoV-19/Poland/PL_ P3794/2021; Lane 14: sample hCoV-19/Poland/PL_P3710/2021; Lane 15: sample hCoV-19/Poland/PL_P3709/2021; Lane 16: SARS-CoV-2 Delta AY.4 control sample, hCoV-19/Poland/PL_P3707/2021; Lane 17: negative control.

thus it may be assumed that these Delta AY.4 isolates with the 872 nt deletion show similar transmissibility to the parental AY.4 lineage.

\section{Ethical statement}

No ethical approval was required for this study as samples were collected for routine surveillance.

\section{Discussion and conclusions}

SARS-CoV-2 VOCs have appeared in Poland in a pattern similar to that observed in other European countries. From July 2021, the Delta VOC has been the dominant circulating variant. Similar to other RNA viruses, the SARS-CoV-2 genome evolves quite rapidly, indicated by a $1.1 \times 10^{3}$ substitution/site/year mutation rate [6]. Numerous changes within SARS-CoV-2 genomes are continuously reported, from minor SNPs to extensive deletions, which have also been reported for other coronaviruses e.g. Middle East respiratory syndrome coronavirus (MERS-CoV) [7]. Regardless of size, all variations may impair the protein structure and function, which could cause changes in the virus phenotype, infectivity, infectiousness, illness severity, or host immune response. The detected $872 \mathrm{nt}$ deletion leads to complete loss of three accessory proteins encoded by the ORF7a, ORF7b, and ORF8 genes.

The gene encoding accessory protein ORF8 shows high variability, and multiple alterations including SNPS, short indels causing frameshifts and partial or complete gene deletions have been reported to date [8-10]. In recombinant SARS-CoV-1, the truncation of ORF8 
Alignment of the consensus sequences of isolates lacking the 872 nt region to the reference SARS-CoV-2 genomic sequence, Poland, 17 July-11 August $2021(\mathrm{n}=14)$

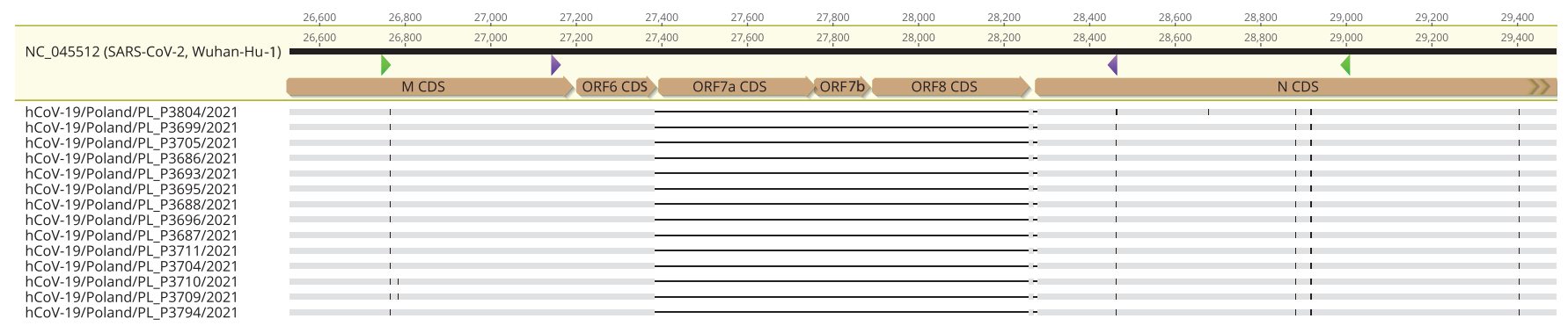

CDS: coding sequence; ORF: open reading frame; SARS-CoV-2: severe acute respiratory syndrome coronavirus 2.

The consensus sequences were combined based on Sanger and Oxford Nanopore Technology sequencing. The triangles marked within the reference sequence (yellow-shaded in the top) represent the primer binding sites of the two primer pairs: purple arrows (90_L and 93_R; ARTICv3 protocol $[4,5])$; green arrows (27_L and 28_R; Midnight protocol [3]).

led to gradual virus attenuation in vitro [7]. In SARSCoV-2, the accessory protein encoded by ORF8 has a function related to evasion of the host adaptive immune response via downregulation of $\mathrm{MHC}-1$ (major histocompatibility complex) [11]. Moreover, ORF8 modulates the host's interferon-mediated antiviral response [12]. However, the importance of ORF8 in vivo remains unresolved. While recombinant $\triangle$ ORF8 SARSCoV-2 viruses produced smaller plaques than the wild type (WT) in vitro, the viral growth kinetics remained unchanged $[10,13]$. Furthermore, human angiotensin converting enzyme 2 (hACE2)-transgenic mice infected with $\triangle O R F 8$ recombinants showed similar pathological lesions and mortality to the WT strain, suggesting that ORF8 does not contribute to virus pathogenicity [13] An observational human cohort study revealed that individuals infected with SARS-CoV-2 lacking a functional ORF8 gene was associated with lower odds of developing hypoxia requiring supplemental oxygen [14].

The deletions within the ORF7 $a$ and $O R F 7 b$ region have also previously been reported [15-18]. ORF7a activates the nuclear factor kappa-light-chain-enhancer of activated B cells (NF-kappa B) pathway and induces proinflammatory cytokine expression $[19,20]$. The function of ORF7b is largely unknown, but a recent study has shown that the protein activates the type-I interferon (IFN) signalling pathway and promotes expression of IFN-beta, interleukin (IL)-6, and tumor necrosis factor (TNF)-alpha, which induce apoptosis [21]. Viruses lacking these genes also produced smaller plaques than WT viruses in vitro, but their growth kinetics were similar to the parental strain [13]. Based on studies in animal models, the proteins have a minor impact on pathological lesions and disease outcomes [13].
This is the first study, to our knowledge, confirming the emergence of such a large deletion within the SARSCoV-2 genome, causing the complete loss of three consecutive ORF sequences, e.g. ORF7a, ORF $7 b$, and ORF8. The clinical data obtained from 25 infected individuals suggest a typical course of COVID-19, with mild to moderate symptoms. The origin of these Delta AY.4 isolates with the $872 \mathrm{nt}$ deletion remains unknown, but their detection in a short period of time with high frequency in the Malopolska voivodship suggests that they may be maintained in the population. Based on GISAID data, during recent weeks (September 2021), more than 70 similar sequences containing a stretch of N's within the region corresponding to ORF7a, ORF $7 b$ and $O R F 8$ were confirmed in other areas in Poland, including Mazowieckie, Pomorskie and Swietokrzyskie voivodships. These sequences, similar to those presented here, could also contain the 872 nt deletion and thus, detection could be missed because of the bioinformatic algorithm leading to a long stretch of N's at the position of the deleted genes. However, a re-analysis of raw sequencing data or Sanger sequencing is needed to confirm the deletion in these samples. Considering the potential spread of a virus strain similar to these Delta AY. 4 isolates with the $872 \mathrm{nt}$ deletion to the other regions in Poland, it may be hypothesised that its transmissibility has not been compromised. Unfortunately, the original swab samples were unavailable and the virus isolation was impossible at this point, but will be a subject for the future studies. The monitoring of this 872 nt deletion in clinical samples and the publicly available databases should be continued. 


\section{FIGURE 3}

Comparison of viral load between the SARS-CoV-2 Delta AY.4 lineage $(n=34)$ and the Delta AY.4 isolates with the 872 nt deletion $(\mathrm{n}=30)$, Poland, 17 July-11 August 2021

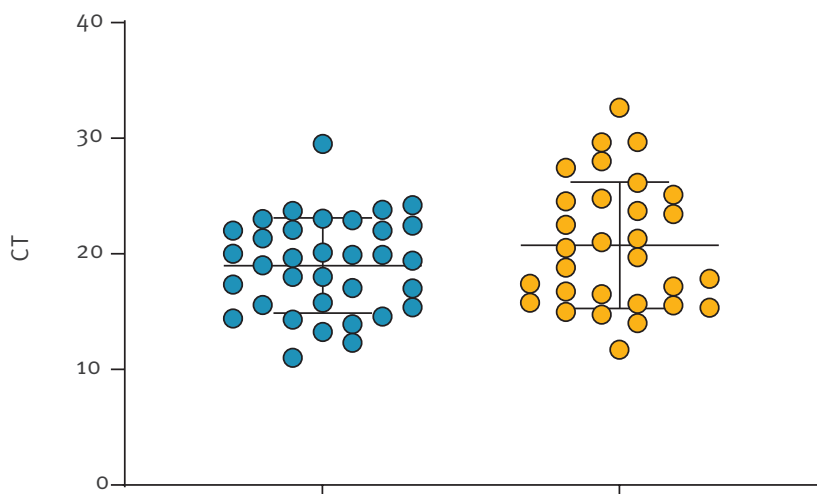

Parental AY.4 lineage

$\triangle 872 \mathrm{nt}$

CT: cycle threshold; SARS-CoV-2: severe acute respiratory syndrome coronavirus 2 .

Each point refers to single sample, for which the CT value was calculated as a mean CT obtained from three target genes $(E, N$, ORF1a) obtained during routine diagnostic RT-qPCR. CT values are compared between SARS-CoV-2 AY.4 samples $(n=34)$ and Delta AY. 4 isolates with the 872 nt deletion $(n=30)$. Error bars indicate standard deviation of the mean. The difference is statistically insignificant ( $p>0.05$, unpaired t-test).

\section{Acknowledgements}

We would like to thank Provincial Sanitary and Epidemiological Station in Krakow for their excellent cooperation and clinical data disclosure.

Funding statement: This work was supported by the subsidy from the Polish Ministry of Science and Higher Education for the research on the SARS-CoV-2 and the grant 2020/ABM/ COVID19/UJ from the Polish Agency of Biomedical Sciences to KP. LR was supported by the Ministry of Science and Higher Education Decision No. 54/WFSN/2020. "Co-infections with SARS-CoV-2, Database of COVID-19 Accompanying Infections".

\section{Conflict of interest}

None declared.

\section{Authors' contributions}

Study design: NMP, LR, KP. Data analysis: NMP, LR, KP. Experimental procedures: NMP, LR, TG, GN, MK, WW, PS, MK, KG, NDM, JG, APM, BS, KBS, JS, MG, PL. Manuscript preparation: NMP, LR, KP. Manuscript revision: all authors.

\section{References}

1. Global Initiative on Sharing All Influenza Data (GISAID). Munich: GISAID. [Accessed: 15 Sep 2021]. Available from: https://www.gisaid.org

2. World Health Organization (WHO). Laboratory testing fo coronavirus disease ([COVID-19) $\square$ in suspected human cases: interim guidance, 19 March 2020. Geneva: WHO; 2020. Available from: https://apps.who.int/iris/handle/10665/331501

3. Freed N, Silander O. SARS-CoV2 genome sequencing protocol (1200bp amplicon "midnight” primer set, using Nanopore Rapid kit). protocols.io. 2021. https://doi.org/10.17504/ protocols.io.bwyppfvn

4. Quick J. nCoV-2019 sequencing protocol v3 (LoCost). protocols.io. 2020. Available from: https://protocols.io/view/ ncov-2019-sequencing-protocol-v3-locost-bh42j8ye

5. Tyson J, James P, Stoddart D, Sparks N, Wickenhagen A, Hall G, et al. Improvements to the ARTIC multiplex PCR method for SARS-CoV-2 genome sequencing using nanopore. bioRxiv 2020.09.04.283077; https://doi. org/10.1101/2020.09.04.283077

6. Duchene S, Featherstone L, Haritopoulou-Sinanidou M, Rambaut A, Lemey P, Baele G. Temporal signal and the phylodynamic threshold of SARS-CoV-2. Virus Evol. 2020;6(2):veaao61. https://doi.org/10.1093/ve/veaao61 PMID: 33235813

7. Muth D, Corman VM, Roth H, Binger T, Dijkman R, Gottula LT, et al. Attenuation of replication by a 29 nucleotide deletion in SARS-coronavirus acquired during the early stages of humanto-human transmission. Sci Rep. 2018;8(1):15177. https://doi. org/10.1038/s41598-018-33487-8 PMID: 30310104

8. Pancer K, Milewska A, Owczarek K, Dabrowska A, Kowalski M, Łabaj PP, et al. The SARS-CoV-2 ORF10 is not essential in vitro or in vivo in humans. PLoS Pathog. 2020;16(12):e1008959. https://doi.org/10.1371/journal.ppat.1008959 PMID: 33301543

9. Pereira F. Evolutionary dynamics of the SARS-CoV-2 ORF8 accessory gene. Infect Genet Evol. 2020;85:104525. https:// doi.org/10.1016/j.meegid.2020.104525 PMID: 32890763

10. Gamage AM, Tan KS, Chan WOY, Liu J, Tan CW, Ong YK, et al. Infection of human nasal epithelial cells with SARS-CoV-2 and a 382-nt deletion isolate lacking ORF8 reveals similar viral kinetics and host transcriptional profiles. PLoS Pathog. 2020;16(12):e1009130. https://doi.org/10.1371/journal. ppat.1009130 PMID: 33284849

11. Zhang Y, Chen Y, Li Y, Huang F, Luo B, Yuan Y, et al. The ORF8 protein of SARS-CoV-2 mediates immune evasion through down-regulating MHC-I. Proc Natl Acad Sci USA. 2021;118(23):e2024202118. https://doi.org/10.1073/ pnas.2024202118 PMID: 34021074

12. Li J-Y, Liao C-H, Wang O, Tan Y-J, Luo R, Oiu Y, et al. The ORF6, ORF8 and nucleocapsid proteins of SARS-CoV-2 inhibit type I interferon signaling pathway. Virus Res. 2020;286:198074. https://doi.org/10.1016/j.virusres.2020.198074 PMID: 32589897

13. Silvas JA, Vasquez DM, Park J-G, Chiem K, Allué-Guardia A, Garcia-Vilanova A, et al. Contribution of SARS-CoV-2 accessory proteins to viral pathogenicity in K18 human ACE2 transgenic mice. J Virol. 2021;95(17):e0040221. https://doi.org/10.1128/ JVI.00402-21 PMID: 34133899

14. Young BE, Fong SW, Chan YH, Mak TM, Ang LW, Anderson DE, et al. Effects of a major deletion in the SARS-CoV-2 genome on the severity of infection and the inflammatory response: an observational cohort study. Lancet. 2020;396(10251):60311. https://doi.org/10.1016/S0140-6736(20)31757-8 PMID: 32822564

15. Panzera Y, Ramos N, Frabasile S, Calleros L, Marandino A, Tomás G, et al. A deletion in SARS-CoV-2 ORF7 identified in COVID-19 outbreak in Uruguay. Transbound Emerg Dis. 2021;10.1111. https://doi.org/10.1111/tbed.14002 PMID: 33501730

16. Tse H, Wong SC-Y, Ip K-F, Cheng VC-C, To KK-W, Lung DC, et al. Genome sequences of three SARS-CoV-2 ORF7a deletion variants obtained from patients in Hong Kong. Microbio Resour Announc. 2021;10(15):7-9. https://doi.org/10.1128/ MRA.00251-21 PMID: 33858933

17. Addetia A, Xie H, Roychoudhury P, Shrestha L, Loprieno M, Huang $M-L$, et al. Identification of multiple large deletions in ORF7a resulting in in-frame gene fusions in clinical SARS CoV-2 isolates. J Clin Virol. 2020;129:104523. https://doi. org/10.1016/j.jcv.2020.104523 PMID: 32623351

18. Joonlasak K, Batty EM, Kochakarn T, Panthan B, Kümpornsin $\mathrm{K}$, Jiaranai P, et al. Genomic surveillance of SARS-CoV-2 in Thailand reveals mixed imported populations, a local lineage expansion and a virus with truncated ORF7a. Virus Res. 2021;292:198233. https://doi.org/10.1016/j. virusres.2020.198233 PMID: 33227343

19. Su CM, Wang L, Yoo D. Activation of NF-kB and induction of proinflammatory cytokine expressions mediated by ORF7a protein of SARS-CoV-2. Sci Rep. 2021;11(1):13464. https://doi. org/10.1038/s41598-021-92941-2 PMID: 34188167

20. Zhou Z, Huang C, Zhou Z, Huang Z, Su L, Kang S, Chen X, Chen Q, He S, Rong X, Xiao F, Chen J, Chen S. Structural insight reveals SARS-CoV-2 ORF7a as an immunomodulating factor 
for human CD14+ monocytes. iScience. 2021;24(3):102187.

https://doi.org/10.1016/j.isci.2021.102187 PMID: 33615195

21. Yang R, Zhao Q, Rao J, Zeng F, Yuan S, Ji M, et al. SARS-CoV-2 Accessory protein ORF7b mediates Tumor Necrosis Factor-a-

induced apoptosis in cells. Front Microbiol. 2021;12:654709.

https://doi.org/10.3389/fmicb.2021.654709 PMID: 34484133

\section{License, supplementary material and copyright}

This is an open-access article distributed under the terms of the Creative Commons Attribution (CC BY 4.0) Licence. You may share and adapt the material, but must give appropriate credit to the source, provide a link to the licence and indicate if changes were made.

Any supplementary material referenced in the article can be found in the online version.

This article is copyright of the authors or their affiliated institutions, 2021. 